\title{
The "Open" Hopkinson Pressure Bar: Towards Addressing Force Equilibrium in Specimens with Non-uniform Deformation
}

\author{
R. A. Govender ${ }^{1} \cdot$ R. J. Curry ${ }^{1}$
}

Received: 31 August 2015/ Accepted: 8 December 2015/Published online: 29 December 2015

(C) Society for Experimental Mechanics, Inc 2015

\begin{abstract}
Conventional Split Hopkinson Pressure Bar (SHPB) testing requires the verification of force equilibrium in a specimen for the test data to be considered valid. For very low impedance materials the large impedance mismatch between input bar and specimens leads to significant uncertainty in the force measurement at the input face. This makes it difficult to fulfil the equilibrium requirement for conventional SHPB testing of very low impedance materials. Cellular materials further complicate matters, as non-uniform densification can lead to different stress states on either side of a densification front. A novel configuration, termed the Open Hopkinson Pressure Bar (OHPB), is proposed to address the difficulties in measuring small differences in forces on either side of the specimen. The specimen is placed on a HPB, and impacted directly by an instrumented striker (effectively another HPB). This arrangement only requires the processing of one wave in each bar, as opposed to the three waves required in a conventional SHPB. This technique allows significant improvements to be made in the resolution of the force measurements.
\end{abstract}

Keywords Hopkinson pressure bar - Direct impact . Cellular material

R. A. Govender

reuben.govender@uct.ac.za

1 Blast Impact and Survivability Research Unit (BISRU), Mechanical Engineering University of Cape Town, Private Bag X3, Rondebosch 7701, South Africa

\section{Introduction}

Split Hopkinson pressure bar (SHPB) testing requires that both faces of the specimen have approximately equal forces (quasi-equilibrium) in order to obtain a valid stressstrain relationship from the test. Difficulties in obtaining quasi-equilibrium can complicate the measurement of small strain responses, often leading to test data for particularly brittle specimens being rejected. Pulse shaping or smoothing is often employed to facilitate rapid equilibration of the specimen. However, pulse shaping is generally accompanied by a slower rise in strain rate at the start of loading. The increased rise time of the strain rate may be undesirable for specimens which are particularly strain rate sensitive. Difficulties in obtaining equilibrium are exacerbated by large impedance mismatches between the pressure bars and specimens. SHPB testing of cellular materials such as foams and biological material require careful attention to ensure specimen equilibrium and obtain valid test data. The conventional compressive SHPB, proposed by Kolsky [1], allows the forces and velocities at the faces of the bars to be inferred from strain gauge histories at locations distant from the faces. The force $F_{i}$ and velocity $v_{i}$ of the input bar end are inferred from the measured incident $\left(\varepsilon_{i}\right)$ and reflected $\left(\varepsilon_{r}\right)$ strain waves via:

$$
\begin{aligned}
& F_{i}(t)=A_{b a r} E_{y}\left[\varepsilon_{i}^{\prime}(t)+\varepsilon_{r}^{\prime}(t)\right] \\
& v_{i}(t)=C_{o}\left[\varepsilon_{i}^{\prime}(t)-\varepsilon_{r}^{\prime}(t)\right]
\end{aligned}
$$

The transmitted bar force $F_{t}$ and velocity $v_{t}$ depend only on the transmitted $\left(\varepsilon_{t}\right)$ strain wave:

$$
\begin{aligned}
& F_{t}(t)=A_{b a r} E_{y} \varepsilon_{t}^{\prime}(t) \\
& v_{t}(t) F_{t}(t)=C_{o} \varepsilon_{t}^{\prime}(t)
\end{aligned}
$$


Fig. 1 Schematics of a forward and $\mathbf{b}$ reverse direct impact HPB configuration $\mathbf{c}$ open HPB (a) Forward

(b) Reverse

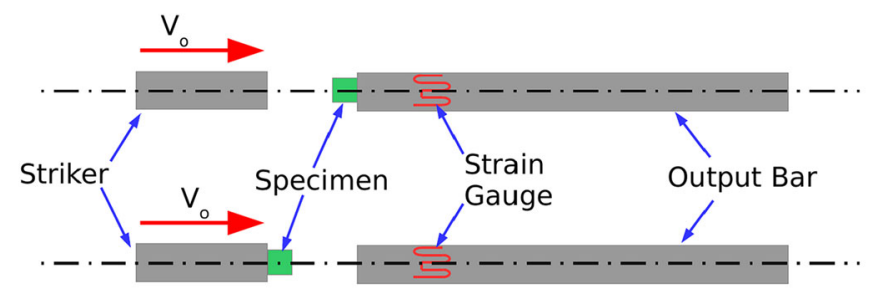

(c) Open

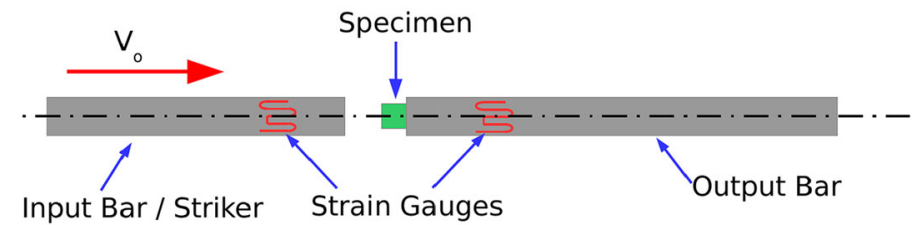

When testing low impedance materials, the magnitude of $\varepsilon_{r}$ is similar to the magnitude of $\varepsilon_{i}$ due to the impedance mismatch between bar and specimen. The data acquisition system must have suitable excitation voltages and gains such that the peaks of both of these waves are captured. As $\varepsilon_{i}$ and $\varepsilon_{r}$ have different signs, $F_{i}$ is proportional to the difference in magnitude of $\varepsilon_{i}$ and $\varepsilon_{r}$. This magnitude difference is small in comparison to the size of the signals which are captured, causing the measurement of $F_{i}$ to have relatively poor resolution and significant uncertainty. Furthermore, as the strain gauge on the input bar is at midlength, the incident and reflected waves have propagated significant distances and thus feature oscillations which arise from geometric dispersion. The magnitude of $\varepsilon_{t}$ is also small in comparison to $\varepsilon_{i}$, as $\varepsilon_{t}$ is limited by the strength of the specimen. Unless the data acquisition system is set with a different gain or excitation voltage for the transmitted bar, this also means the measurement of $F_{t}$ has poor resolution. A typical example of this may be seen in Tagarielli et al. [2], where $F_{i}$ initially overshoots before oscillating about $F_{t}$. The use of semi-conductor strain gauges on metallic bars does improve the signal-noise ratio. However, if the strains in the HPB are so small that semi-conductor gauges are warranted, the waves are likely to be more sensitive to external perturbations such as misalignment or friction in the bar supports.

Cellular materials are also prone to non-uniform deformation and densification as the rate of loading increases [3]. A cellular material which is deforming non-uniformly develops non-uniform density. As specimen density affects both strength and wave propagation, it is expected that the instantaneous forces on the impacted and distal faces of the specimen would differ, both during initial loading and if a densification front develops. It is extremely unlikely that these differences could be resolved from a conventional SHPB configuration, due to the uncertainties described above. Tagarielli et al. [2] stated that for a typical PVC foam, the effects of non-uniform densification had a significant effect on crush strength for compression velocities of approximately $60 \mathrm{~ms}^{-1}$. Impacting a HPB with a striker of identical material and diameter at $60 \mathrm{~ms}^{-1}$ results in a bar stress of $1200 \mathrm{MPa}$ for a steel HPB and 400 $\mathrm{MPa}$ for an aluminium bar, assuming one dimensional elastic wave theory. Hence the loading velocities required to obtain significant non-uniform deformation effects would require metallic HPB of exotic alloys to ensure the bars remained elastic.

Deshpande and Fleck [4] proposed the use of both forward and reverse direct impact HPB tests (Fig. 1) to measure the specimen stress on either side of a densification front in cellular materials. In the conventional forward Direct Impact HPB test, the specimen is mounted on the HPB and impact is between the specimen and striker. The output bar therefore measures the stress at the distal (nonimpacted) face. In reverse Direct Impact HPB tests, the specimen is mounted to the striker and both striker and specimen are accelerated towards the output bar. As impact is between the specimen and output bar, the stress measured is that of the proximal (impacted) face. Radford et al. [5] used a configuration similar to the Reverse Direct Impact HPB, where Alporas aluminium foam specimens were fired directly against a HPB. This arrangement is effectively a Taylor cylinder impact test, where the HPB provides a force or stress history.

The direct impact HPB has several advantages: higher impact velocities than the SHPB are possible; and the output bar properties and data acquisition chain may be optimised to give well resolved signals. As the force and velocity history is only available for one specimen face, it is impossible to verify equilibrium. Furthermore, different specimens must be used for each of the forward and reverse tests. Hence any normal variation between specimens will result in a measurable difference between forward and reverse tests, potentially obscuring the phenomena of nonuniform densification. Meenken and Hiermaier [6, 7] used a direct impact HPB, where both the striker and HPB were 


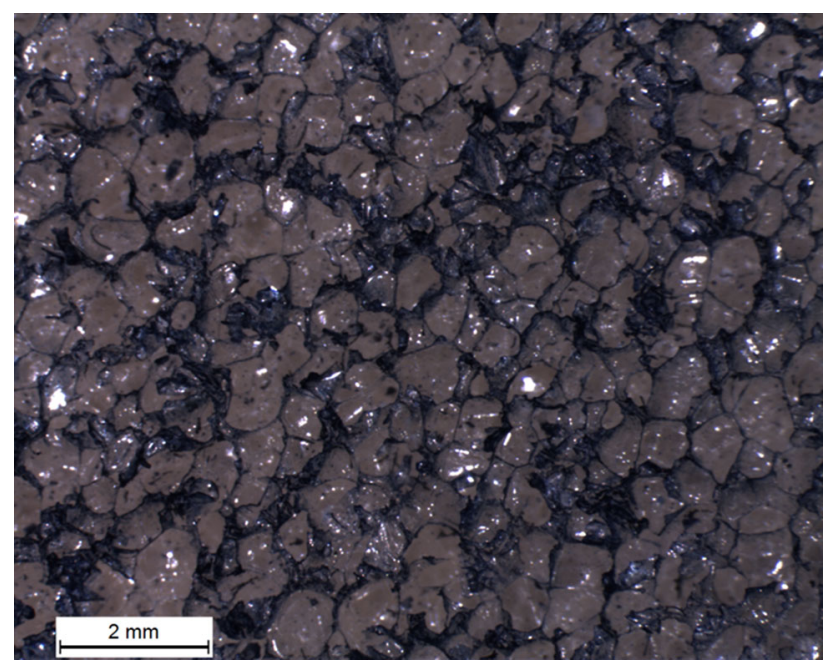

Fig. 2 Typical cellular structure of Corecell A500 (raised walls coloured to aid in contrast for cell size estimation)

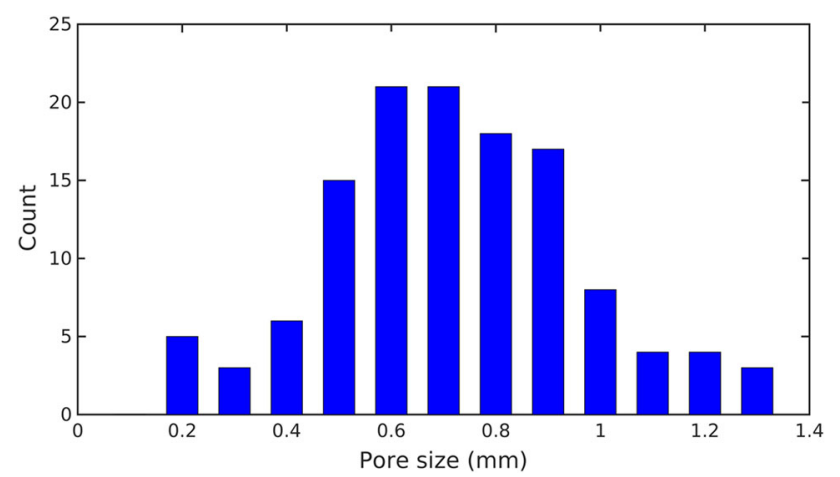

Fig. 3 Pore size distribution of a typical Corecell A500 sample

instrumented with PVDF film gauges, to investigate large deformations of soft materials such as rubber. This configuration provided well resolved stress measurements on both specimen faces, but requires charge amplifiers for the PVDF gauges which are not as commonly available as the strain gauge amplifiers used in most HPB experiments. The arrangement appeared to have difficulties beyond the peak load of the experiment once the specimen began to relax. This was attributed to the PVDF gauges being mounted without any preload. An alternative to PVDF gauges is to use Doppler velocimetry to measure the stress waves in the striker in a non-contact manner. Doppler velocimetry was applied to SHPB testing by Casem and Zellner [8] and to Direct Impact HPB testing by Lea and Jardine [9]. While Doppler velocimetry is promising, the necessary instrumentation was not available to the authors.

This paper presents a novel configuration for a HPB experiment, which permits the measurement of the forces on both faces of the specimen with significantly improved resolution, using only the strain gauge instrumentation already used in all modern HPB arrangements.

\section{Material and Experimental Details}

The material investigated was styrene acrylonitrile (SAN) foam (Corecell A500), which has a nominal density of 92 $\mathrm{kg} \mathrm{m}^{-3}$. Specimens of nominal diameter $18 \mathrm{~mm}$ and two lengths (10 and $20 \mathrm{~mm}$ ) were used. A typical cross section of the SAN foam is shown in Fig. 2. The mean pore size was found to be $0.7 \mathrm{~mm}$ using the line-intercept method, with the distribution of pore sizes shown in Fig. 3. The specimens have an average of 25 cells across the diameter. Previous investigations of polymeric foams using the SHPB have utilised specimens with average cell count across the diameter ranging from 19 [10] to 36 [2]. Quasistatic compression tests were conducted at a nominal strain rate of $1.6 \times 10^{-3} \mathrm{~s}^{-3}$. The specimen faces were lubricated with grease for all tests.

\section{SHPB Tests}

Conventional compression SHPB tests were conducted, using $20 \mathrm{~mm}$ diameter PMMA bars. PMMA was selected to reduce the impedance mismatch between specimens and bars, in order to improve the signal-noise ratio. The usual SHPB relationships (Eqs. 2, 4) are utilised, but the shifting of the waves from the strain gauges to the bar ends must account for attentuation and dispersion effects. The viscoelastic wave propagation in the PMMA bars was characterised using the technique described by Bacon [11]. A wave measured at any location $z_{0}$ may be shifted to any other location $z_{1}$ by means of a propagation coefficient $\gamma(\omega)$ in the frequency domain:

$\varepsilon^{\prime}\left(\omega, z_{1}\right)=\varepsilon\left(\omega, z_{0}\right) e^{\gamma(\omega)\left(z_{1}-z_{0}\right)}$

The propagation coefficient corrects for both attenuation $\alpha(\omega)$ and wave speed (dispersion) $C(\omega)$ effects:

$\gamma(\omega)=\alpha(\omega)+\frac{i \omega}{C(\omega)}$

The relationship between propagation coefficient $\gamma(\omega)$ and complex modulus $E^{*}(\omega)$ is given by:

$\gamma^{2}(\omega)=\frac{-\rho \omega^{2}}{E^{*}(\omega)}$

The complex modulus relates stress and strain in the frequency domain:

$\sigma(\omega)=E^{*}(\omega) \varepsilon(\omega)$

$\alpha(\omega)$ and $C(\omega)$ are characterised experimentally by measuring wave propagation in a free HPB without a specimen, and exploiting the fact that the distal bar end must be stress free. The incident and reflected waves are transformed from the time domain $\left(\varepsilon_{i}(t), \varepsilon_{r}(t)\right)$ to the frequency domain 
$\left(\varepsilon_{i}(\omega), \varepsilon_{r}(\omega)\right)$ using the Fourier transform. The attenuation function $\alpha(\omega)$ is derived from the logarithmic ratio of amplitudes of the incident and reflected waves at any given frequency, given the distance from the gauge to the free surface $Z_{g}$ :

$\alpha(\omega)=-\frac{1}{2 Z_{g}} \ln \left(\frac{\left|\varepsilon_{r}(\omega)\right|}{\left|\varepsilon_{i}(\omega)\right|}\right)$

The wave speed function $C(\omega)$ is derived by considering the phase shift between incident and reflected waves at any given frequency. The unwrapped phase angles of each frequency component $\theta_{i}(\omega)$ and $\theta_{r}(\omega)$ are computed. The wave number $k(\omega)$ is related to the phase shift from the incident wave to the reflected wave by:

$k(\omega)=-\frac{\theta_{r}(\omega)-\theta_{i}(\omega)}{2 Z_{g}}$

$C(\omega)$ is related to wave number by:

$C(\omega)=\frac{\omega}{k(\omega)}$

Further details of this procedure are found in Bacon and Curry et al. [11, 12]. The PMMA bars have a density of $\rho=1195 \mathrm{~kg} \mathrm{~m}^{-3}$ and a fundamental wave speed $C_{0}=2160 \mathrm{~m} \mathrm{~s}^{-1}$. The data acquisition system used in the wave characterisation and SHPB tests has a 16 bit resolution and sampled each channel at $10 \mathrm{MSa} / \mathrm{s}$. For the SHPB tests, the bridge excitation voltage was set to $1.0 \mathrm{~V}$.

\section{Open Hopkinson Pressure Bar Configuration}

The configuration under development in this investigation is termed the Open Hopkinson Pressure Bar (OHPB). It is essentially a Direct Impact HPB configuration, where the striker is instrumented with strain gauges, as shown in Fig. 1c. A significant difference from other direct impact HPB arrangements is that a much longer striker $(\sim 1200 \mathrm{~mm})$ is used. If the instrumented striker (input bar) were shorter, the measurement duration would be limited. The output bar is $\sim 2000 \mathrm{~mm}$ in length, while both bars have a diameter of $20 \mathrm{~mm}$. The striker is supported in an oversize barrel $(36 \mathrm{~mm})$ by polymer bushings, which primarily allow room for the strain gauge cables, but also permit greater acceleration for the same gas pressure due to the increased area. The striker is accelerated towards the specimen and output bar using a conventional gas gun, and its velocity just prior to impact is measured using a light trap. In this arrangement, the stress waves originate at the specimen face impacted by the striker, and propagate away from the specimen in both bars. This is important as only one wave in each bar is required to infer the force and velocity at the specimen end of that bar. A conventional
SHPB requires two waves (incident and reflected) to calculate the force and velocity of the input bar face. This implies that the initial, unloaded strain gauge output voltage be set at the mid-point of the data acquisition system, with half the system's dynamic range used for the input wave and the other half for the reflected wave. As the Open HPB arrangement requires only a single wave from both the input and output bar, of practically equal magnitude, the amplification and data acquisition system may be adjusted to maximise the resolution of this wave. A further gain is easily obtained by offsetting the unloaded strain gauge output voltage towards the appropriate limit of the data acquisition system prior to the test. This allows almost the entire dynamic range of the data acquisition to be used in capturing the single wave of interest, in comparison to a conventional SHPB where half the dynamic range is allotted to each wave. As with most Direct Impact HPB, the strain gauges may be located closer to the impact faces (300 mm), which allows a longer duration of impact to be captured for the same length of bar. This configuration also allows both input and output bar diameter and material to be tailored to the expected stresses for a given specimen. In this case, PMMA bars similar to those used in the SHPB experiments were employed, with strain gauge locations as noted above and the bridge excitation voltage set to $4.0 \mathrm{~V}$.

In the OHPB, the relationships for the force at the input and output specimen faces reduce to:

$$
\begin{aligned}
& F_{\text {In }}(t)=A_{\text {In }} E_{y} \varepsilon_{\text {In }}^{\prime}(t) \\
& F_{\text {Out }}(t)=A_{\text {Out }} E_{y} \varepsilon_{\text {Out }}^{\prime}(t)
\end{aligned}
$$

Given the original specimen length $l_{0}$ and striker velocity $V_{0}$, the instantaneous length of the specimen $l_{s}(t)$ is obtained via:

$$
l_{s}(t)=l_{o}-\int_{0}^{t}\left\{V_{o}-C_{o, I n} \varepsilon_{\text {In }}^{\prime}(\tau)-C_{o, \text { Out }} \varepsilon_{\text {Out }}^{\prime}(\tau)\right\} d \tau
$$

As the incompressibility assumption does not hold for cellular materials, all results presented for quasi-static, SHPB and OHPB experiments are engineering stress and strain based on the original specimen area. As high speed video is used to monitor the experiments, it is hoped that calibration and image processing of future tests will enable measurement of the instantaneous radius and hence permit true stress calculation.

\section{Results}

The raw strain gauge voltages from a typical Split HPB test are shown in Fig. 4, while those for a typical Open HPB test are shown in Fig. 5. These signals were both corrected 
for zero-offset in post processing. As the SHPB test was unable to load the specimen to densification, which was possible for the OHPB test, comparisons are only drawn for the plateau stress. For the SHPB test, the average voltage in the plateau region of the transmitted wave is approximately $0.4 \mathrm{~V}$, which is $4 \%$ of the full scale of the data acquisition card. For the OHPB test, this has increased to approximately $1.0 \mathrm{~V}$, corresponding to $10 \%$ of the full scale. This is a useful improvement in resolution. It should be noted that for the OHPB test, the strain gauge bridge voltage was reduced to allow capture of the densification, where the peak voltage is an order of magnitude greater than the plateau. If the OHPB is used to investigate the earlier part of the plateau stress, and the densification data may be foregone, the strain gauge bridge voltage could be increased by a factor of four. This would further improve the resolution of the OPHB data in the plateau region.

Due to the length of the input (instrumented striker) bar in the OHPB test, the data is only sensible up to $1 \mathrm{~ms}$ after impact, after which the reflected waves are superimposed. A small oscillation is visible on the output bar signal at approximately $4.7 \mathrm{~ms}$. This corresponds to the time taken from impact for a wave to travel the entire length of the striker and reflect back to the impact face.

The stress histories, shifted to the specimen faces and corrrected for dispersion and attenuation, are shown in Fig. 6 for the SHPB test, and Fig. 7 for the OHPB test. It is in this comparison that the usefulness of the OHPB arrangement becomes clear. In the SHPB test, the input face stress has oscillations of significant magnitude in comparison to the plateau stress. The output face stress is much more stable, with a clear transition from the initial elastic response to the plateau stress. The input face oscillations reduce certainty in the verification of specimen equilibrium. The striker face stress of the OHPB test (analagous to the input face in the SHPB test) has some oscillations but these are significantly smaller. In the OHPB test, dynamic equilibrium was achieved by a strain of 0.05 , which is within the initial elastic deformation! The

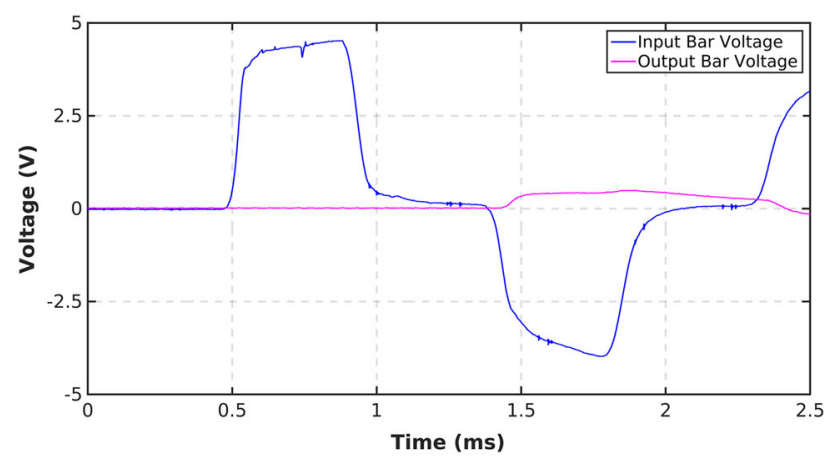

Fig. 4 Strain gauge signals from conventional SHPB test

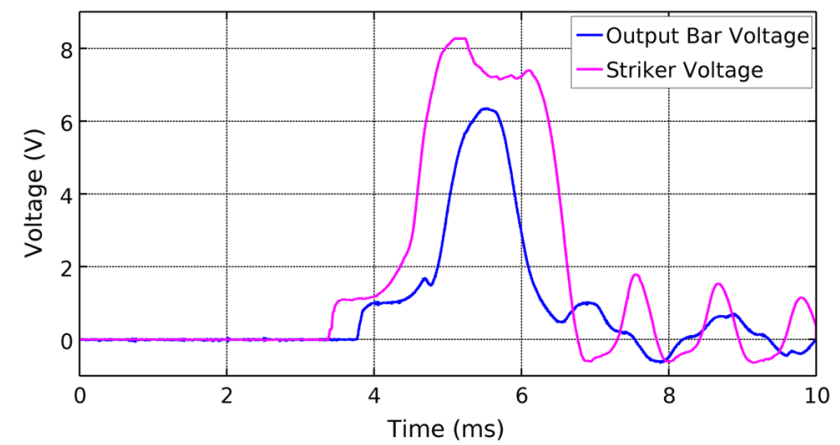

Fig. 5 Strain gauge signals from OHPB test

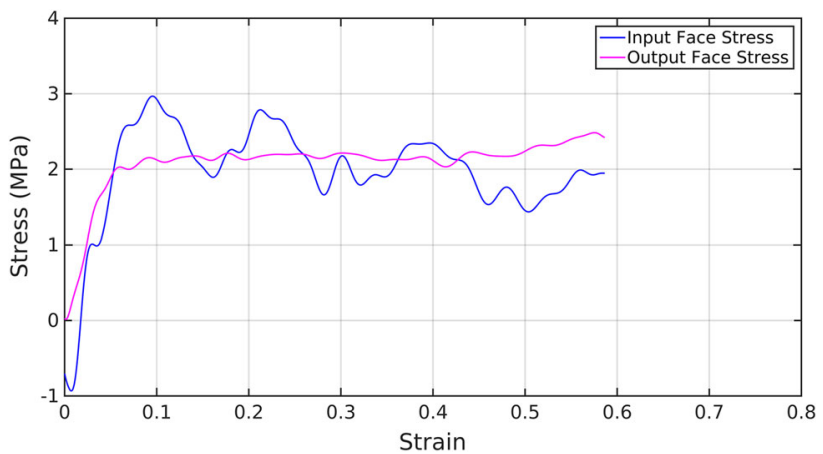

Fig. 6 Split HPB specimen stress versus strain at striker and output faces

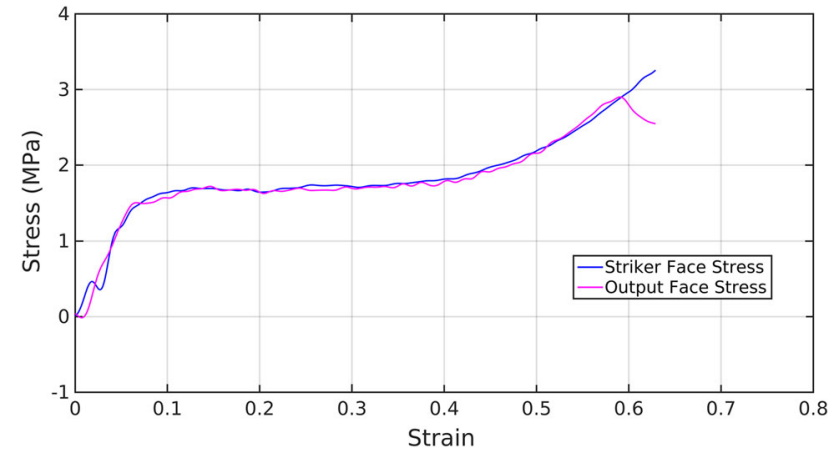

Fig. 7 Open HPB Specimen stress versus strain at striker and output faces

transition to the plateau is well defined for both striker and output faces, and dynamic equilibrium is maintained until the arrival of the reflected wave in the striker. In order to determine elastic modulus from these measurements, it would be necessary to determine the uniformity of specimen strain prior to the stress plateau. This could be facilitated via DIC or similar full field displacement measurements in future testing.

The engineering stress and strain rate histories for the OHPB test are shown in Fig. 10. Due to the direct impact nature of the experiment, the strain rate does not have a 


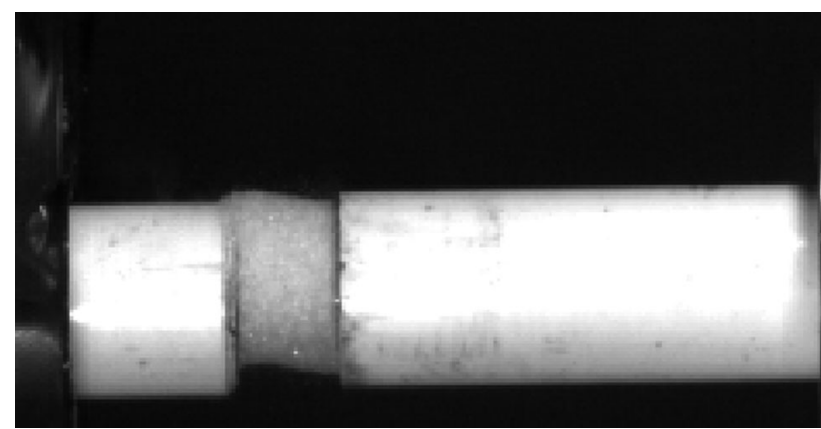

Fig. 8 Frame from test with poor striker and bar alignment

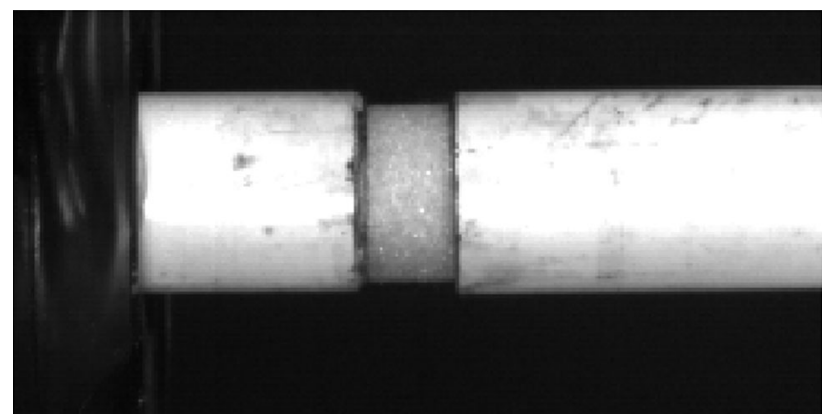

Fig. 9 Frame from test with good striker and bar alignment

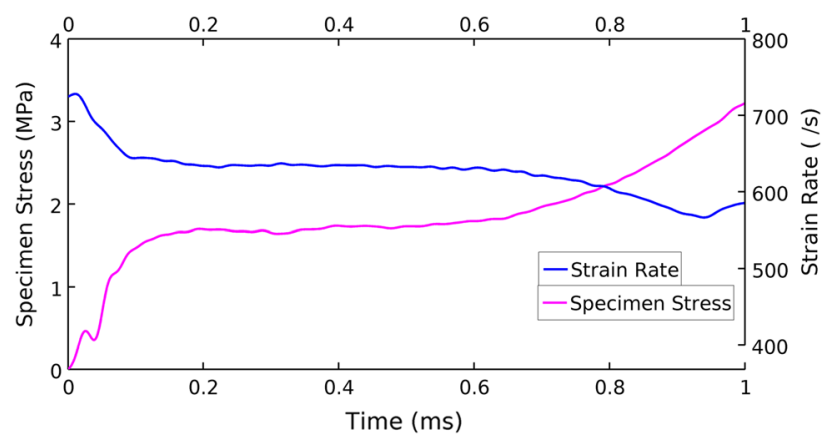

Fig. 10 Engineering stress and strain rate as a function of strain from an OHPB test

"rise time". The average strain was approximately $650 \mathrm{~s}^{-1}$, for a striker velocity of $7.6 \mathrm{~m} \mathrm{~s}^{-1}$. Tests at striker velocities of $9-10 \mathrm{~m} \mathrm{~s}^{-1}$ produced comparable data at a slightly higher strain rate. Unfortunately tests at higher striker velocities encountered difficulties in maintaining strikerspecimen alignment. High speed video was used to monitor all tests, and any tests where the striker-specimen alignment was poor were excluded from further analysis. An example of very poor alignment is shown in Fig. 8. The axes of the output bar and striker were eccentric by approximately $1 \mathrm{~mm}$, leading to a shear deformation of the specimen. Figure 9 shows a test where correct alignment was achieved, and the specimen undergoes uniform compression over its cross-section with no shear component. Acceptable alignment was deemed to be an eccentricity of less than $0.5 \mathrm{~mm}$ of the striker and output bar.

The results of the quasi-static, SHPB and OHPB tests are presented in Fig. 11. The results of the OHPB tests are consistent with those of the SHPB tests. There is a difference in plateau stress measured in the OHPB and SHPB tests. There are two factors which are likely contributors to this difference. Firstly, different pairs of bars were used for the OHPB and SHPB tests. While the calibration of the strain gauge levels was confirmed within each pair, this was not confirmed across the pairs. Hence there is possibly a small difference in strain gauge calibration leading to a corresponding difference in measured stress. Secondly, the OHPB tests were conducted at a lower strain rate than the SHPB tests $\left(6.5 \approx 8.5 \times 10^{2} \mathrm{~s}^{-1}\right.$ in comparison to $1.1 \times$ $10^{3} \mathrm{~s}^{-1}$ ), which could also contribute towards the lower plateau stress.

\section{Discussion and Concluding Remarks}

In the early stress histories of the OHPB tests, a consistent delay between the stress rise of the input and output bar histories is visible, as shown in Fig. 12. This delay may be

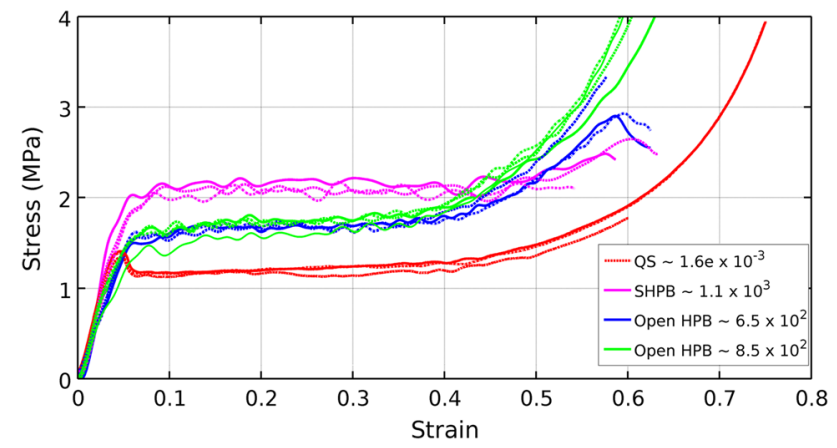

Fig. 11 Collated stress-strain data for quasi-static, SHPB and OHPB tests

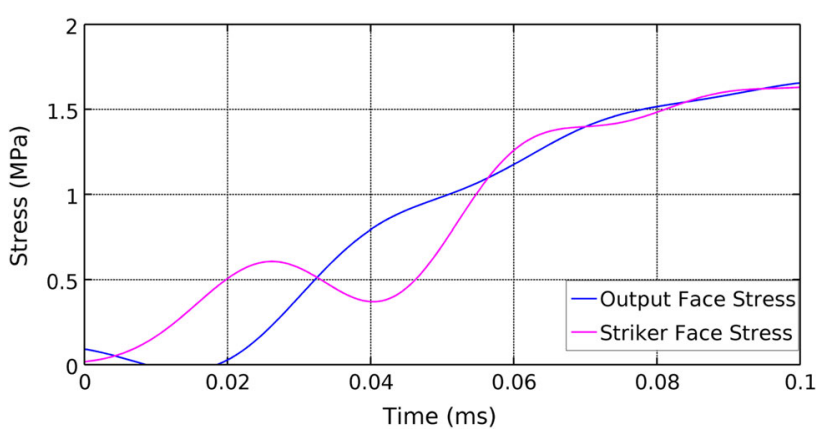

Fig. 12 Detail of the initial stress histories of an OHPB test 
attributed to the fact that deformation begins at the input face and the wave must propagate through the specimen before the output bar is loaded. Across 8 tests, this delay ranged from $15-19 \mu \mathrm{s}$. This gives a nominal wave speed of $C_{0} \approx 520 \sim 670 \mathrm{~m} \mathrm{~s}^{-1}$, which corresponds to a nominal elastic modulus of 27-45 MPa. As the modulus measured from quasi static compression tests was 30-35 $\mathrm{MPa}$, it appears that the delay is consistent with an elastic compression wave propagating from the input (impact) face to the output face of the specimen. This suggests that the OHPB arrangement is able to discern differences at small deformations, which are usually uncertain in conventional SHPB experiments. As noted earlier, testing at higher striker velocities struggled to maintain acceptable alignment. Further development of the OHPB arrangement is currently underway to resolve these issues. The OHPB has proved capable of measuring stress histories on both faces of a specimen with substantially higher resolution than a conventional SHPB arrangement. Increases in impact velocities, coupled with testing a variety of specimen lengths, will hopefully lead to non-uniform deformation of specimens and an opportunity to investigate simultaneous stress histories on both sides of a densification front.

Acknowledgments The financial support of the South African National Research Foundation, via Thuthuka Grant TTK13062019477, is gratefully acknowledged. Dr. Govender extends his thanks to Prof. Gerald Nurick, for his support, guidance and mentorship as well as proofreading and comments on an early version of this article.

\section{References}

1. Kolsky H (1949) An investigation of the mechanical properties of materials at very high rates of loading. Proc Phys Soc Lond B62:676

2. Tagarielli V, Deshpande V, Fleck N (2008) The high strain rate response of PVC foams and end-grain balsa wood. Compos Part B: Eng 39(1):83

3. Merrett R, Langdon G, Theobald M (2013) The blast and impact loading of aluminium foam. Mater Design 44:311

4. Deshpande V, Fleck N (2000) High strain rate compressive behaviour of aluminium alloy foams. Int J Impact Eng 24(3):277

5. Radford D, Deshpande V, Fleck N (2005) The use of metal foam projectiles to simulate shock loading on a structure. Int $\mathrm{J}$ Impact Eng 31(9):1152

6. Meenken T, Hiermaier S (2006) Large strain dynamic compression for soft materials using a direct impact experiment. Phys J IV France 134:653

7. Hiermaier S, Meenken T (2010) Characterization of low-impedance materials at elevated strain rates. J Strain Anal Eng 45:401

8. Casem D, Zellner M (2013) Kolsky bar wave separation using a photon doppler velocimeter. Exp Mech 53(8):1467

9. Lea L, Jardine A (2015) Two-wave photon Doppler velocimetry measurements in direct impact Hopkinson pressure bar experiments. EPJ Web Conf 94:01063

10. Subhash G, Liu Q (2009) Quasistatic and dynamic crushability of polymeric foams in rigid confinement. Int $J$ Impact Eng 36(1011): 1303

11. Bacon C (1998) An experimental method for considering dispersion and attenuation in a viscoelastic Hopkinson bar. Exp Mech 38:242

12. Curry R, Cloete T, Govender R (2012) Implementation of viscoelastic Hopkinson bars. EPJ Web Conf 26:01044 